\title{
Noise Reduction in Shuttle Loom
}

\author{
R.Sukanya Devi ${ }^{1} \mid$ S.L.Jhakan $\operatorname{Prashad}^{2} \mid$ P.Kuhan ${ }^{3}$
}

${ }^{1}$ Assistant Professor, Department of Textile Technology, Kumaraguru College of Technology, Coimbatore, Tamil nadu, India.

${ }^{2}$ Student, Department of Textile Technology, Kumaraguru College of Technology, Coimbatore, Tamil nadu, India.

${ }^{3}$ Student, Department of Textile Technology, Kumaraguru College of Technology, Coimbatore, Tamil nadu, India.

\section{To Cite this Article}

R.Sukanya Devi, S.L.Jhakan Prashad and P.Kuhan, "Noise Reduction in Shuttle Loom", International Journal for Modern Trends in Science and Technology, 6(9S): 132-135, 2020.

\section{Article Info}

Received on 25-August-2020, Revised on 08-September-2020, Accepted on 12-September-2020, Published on 18-September-2020.

\section{ABSTRACT}

Noise pollution is the one of the major problems in our country. Unfortunately, textile field contributes to it, the sound from the shuttle loom is around 102 decibels. This is the major issue for people working in weaving factory and also for people who lives near to it. The consequences of this issue are very serious like people suffer from hearing related diseases due to the noise from shuttle looms. This paper explains various materials and their properties which can be used as the sound reduction material. Finally, this paper discusses various ways to reduce the noise produced from shuttle loom.

Keywords: Noise reduction, Shuttle Looms, Power looms, Sorbothane, Sound reduction, Picker, Vibration dampening.

\section{INTRODUCTION}

Noise is an unwanted sound that interferes with the function in a given space. It is subjective because what is disturbing and unacceptable to one may be acceptable to another. In recent years even, a developing country like India has taken positive steps against excessive noise. Much discussion and legislation has been evolved in an attempt to recognize and combat the problem of noise pollution. Today, the noise produced from the shuttle looms is enormous and people are suffering from various difficulties even in some cases there is a chance for loss of hearing due the noise produced from the looms. Then, too, there is a published general survey of noise ${ }^{[1]}$. This paper explains about various materials we can use for the noise reduction. For this purpose, the operation was to be carried out in a perfect environment. Many investigation have been done to reduce the noise in shuttle weaving by remodeling the looms and by enclosing it in a sound proof cover ${ }^{[2]}$. The whole operation of shuttle looms is carried out. "Whole
Operation" means without the vertical motion of shuttle boxes, healds or warp yarns and with an empty shuttle having no weft yarns in it.

\section{PROCEDURE OF NOISE REDUCTION}

Force produce in the machines causes the noise majorly, this can be overcome by reducing either one or both mass and acceleration. This lead us into various other form of weaving types where the weft insertion is replaced by other means.Crank gears are mostly used nowadays instead of other gears because they give even transmission. There are two possible ways to reduce the noise, one way involves optimum changes in machineries in electro-mechanical way. This could possibly lead us to ANC. ${ }^{[3]}$ ANC, Active Noise Control means sound field modification by electro-acoustical means. Here, an opposite sound is produced for the sound being produced in the machine. So, that both of them nullify each other. But this method is much more complicated. The second way is kind of a passive one. Passive Measures are the way that 
involves not much of a electrical and mechanical systems. Ear protectors are the best examples for a passive method, they are currently using in the industry. One way of doing this is complete enclosure of the machine. But this can be problematic in our shuttle looms and also not a feasible solution. So, the vibration dampening method can be suggested. This involves reduction of natural frequency of vibration and resonance. The best among the vibration dampening material is discussed below.

\subsection{Sorbothane:}

Sorbothane is a material suggested for sound reduction in loom. Sorbothane is a proprietary, visco-elastic polymer. Sorbothane is a thermoset, polyether-based, polyurethane material. In addition to being visco-elastic, Sorbothane also has a very high damping coefficient.

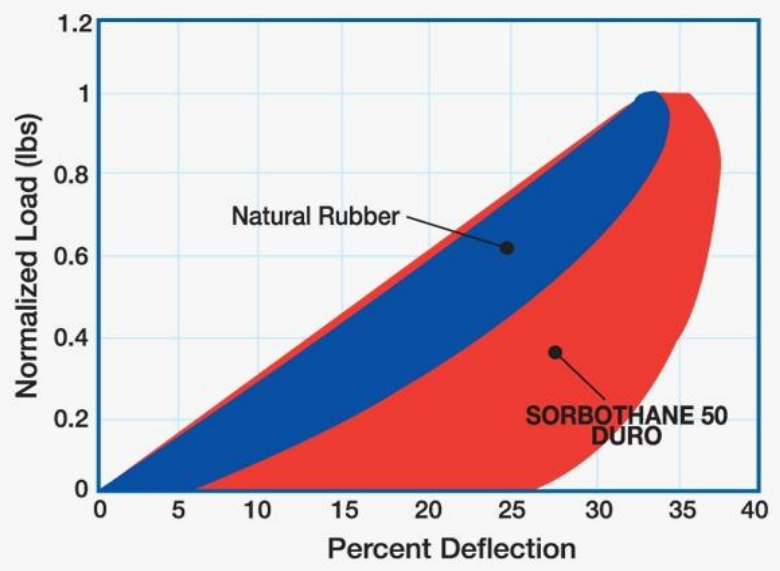

Hysteresis (Mechanical Energy converted to heat each cycle)

Fig.no : 1

Sorbothane combines shock absorption, good memory, vibration isolation and vibration damping characteristics. The one-of-a-kind material Sorbothane has been utilized to create effective vibration absorbing pads to absorb and isolate harmful shock and vibration. Perhaps the shuttle's vibration or shock problem doesn't require a custom solution, but could instead be solved with one of many Standard Industrial Products. Ranging from bumpers to sheet stock and everything in between, the standard products are available in a wide array of sizes and quantities, making these products the perfect place to start. Sorbothane is a unique material exhibiting both solid and liquid properties and can be adapted to any number of uses.

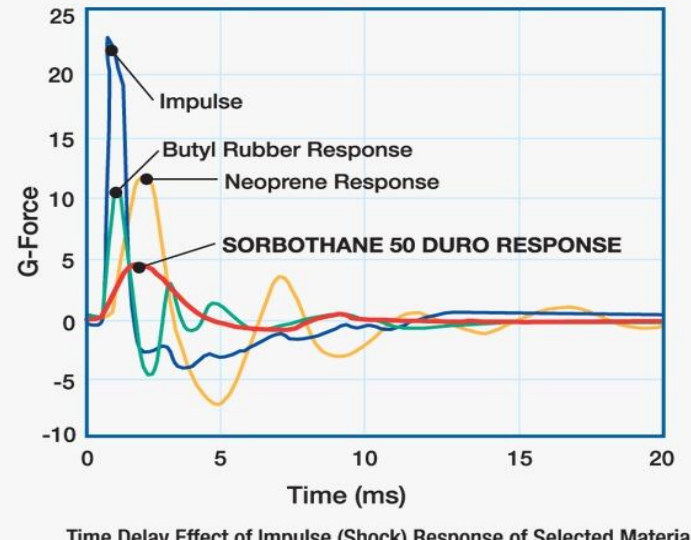

Time Delay Effect of Impulse (Shock) Response of Selected Materials

Fig.no:2

This engineered solution can provide solution for the exact needs in the industry and also provide solutions that will be perfect for the machines. Standard Sorbothane products and custom engineered solutions are available to isolate vibration, noise and attenuate shock which is produced from the machines. Custom components can be specially designed and manufactured to meet the unique properties for noise reduction requirements.

\section{STORAGE CONDITIONS}

In order to obtain the high efficiency, the Sorbothane should be stored in appropriate conditions:

\subsection{Approved storage conditions:}

- Temperature range: 60 to 80 degrees Fahrenheit (16 to 26 degrees Celsius)

- Humidity: 50 percent relative humidity

- Atmospheric conditions: less than $10 \mathrm{ppm}$ of toluene, naphtha, alcohol, gasoline, or other plastic deteriorating solvents

- Material to be stored flat, spaced as shipped and in original packaging and

- Do not stack.

\subsection{Shelf life:}

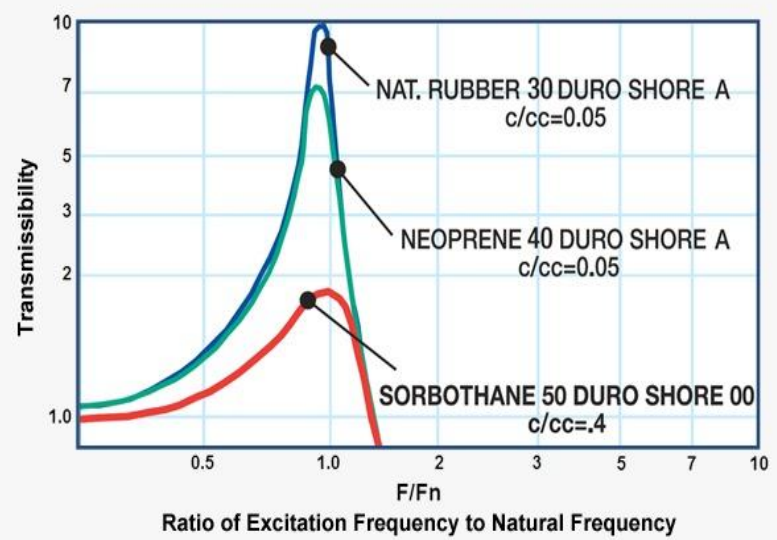

Fig.no:3 
Sorbothane has 3 years from manufactured date if stored under approved storage conditions. For Pressure Sensitive Adhesives (PSA): 24 months from manufactured date if stored under approved storage conditions.

\section{NOISE IN A WEAVING PLANT:}

Sound is transported through a medium like air, water etc. By means of its rarefaction and compression. There is also a published work $^{[4]}$ on noise levels in various kinds of factories. The energy transmitted in the process per unit time by unit area of the compressing and expanding medium provides a measure of the intensity of sound. Since energy per unit time is equivalent to power in Watts, the unit of sound intensity is expressed in watts/ sq.m, m. The sound power of an average whisper is $0.1 \mu \mathrm{W}$. The air pressure fluctuation created by sound is measured in Pascal (Pa). A normal human being can sense sound pressure varying in the range of $20 \mu \mathrm{Pa}$ to $20 \mathrm{~Pa}$. A logarithmic decibel scale $(\mathrm{dB})$ is used to measure sound with reference to hearing ability of human beings. A sound level of OdB is assigned to a sound intensity of $10-12 \mathrm{~W} / \mathrm{sq} . \mathrm{m}$. Tokita's ${ }^{[5]}$ published work of 1963 gave the results of measurements of noise generated by many kinds of machines and suggested ways to control noise generated by some of the machines.

Table no:1Noise levels in various parts of
shuttle looms[3]
\begin{tabular}{|c|c|}
\hline Section & Noise level (dBA) \\
\hline Warping & $80-86$ \\
\hline Sizing & $73-86$ \\
\hline $\begin{array}{c}\text { Loom shed (non } \\
\text { automatic) }\end{array}$ & $94-99$ \\
\hline $\begin{array}{c}\text { Loom shed } \\
\text { (automatic) }\end{array}$ & $95-97$ \\
\hline
\end{tabular}

Expressing sound level in $\mathrm{dB}$ scale is a convenient method of compressing the scale of numbering the scale of number associated with the variation in pressure into a manageable range from $10 \log (100)$ $=0 \mathrm{~dB}$ to $10 \log (1012) \mathrm{dB}=120 \mathrm{~dB}[6]$. The decibel is a dimensionless number, which relates sound intensity or sound pressure levels to some reference point. When most people use the term decibel or discuss noise levels in decibels, they refer to decibels as related to the A-weighted scale (dBA). The A-weighted scale parallels the sensitivity of the human ear and uses the lowest audible sound that the human ear can detect as the reference point for determining the decibel level of a noise.

Any noise rating above $80 \mathrm{dBA}$ produces physiological effects and any long exposure at above 90 or 100 decibels will produce permanent damage to a person's hearing. An increase of 10 $\mathrm{dBA}$ is a doubling of loudness with respect to the human ear.Noise generally consists of many tones with varying rates of vibration or frequency. The frequency, expressed in cycles per sound and referred to as cps or Hertz $(\mathrm{Hz})$, is usually in the range of $20-20,000$ cycles per second. The ear is not very responsive to very low or very high tones as it is selective to the tones of medium frequency. As mentioned earlier, the dBA scale matches the response of the ear and is, therefore, well suited for evaluating noise as it relates to human beings. Excessive noise level of $94-99 \mathrm{dBA}$ is in loom shed, depending upon the design, type, erection and number of looms used, machine footing and foundation, condition of machines, fabric structure, building type, building size, etc.When the loom is run with a shuttle, noise level increases by about $1.5-2.0 \mathrm{dBA}$. The implementation of Sorbothane is done in a way that the vibrations from the shuttle are getting reduced by which we can reduce the noise. Initially, the material is placed in the sides of the picker, so that when the shuttle is hitting the picker the vibrations are halted by Sorbothane. This is the way of implementing the Sorbothane at starting. By studying this way, the Sorbothane can be implemented in other major parts of the looms too.

\section{PROLONGED EXPOSURE OF NOISE:}

The highest noise level in area samples was observed in the weaving section $(99.5 \pm 3.2 \mathrm{dBA})$. History of hearing loss was reported by $8.1 \%$ participants, while $9.0 \%$ reported a history of ear related diseases. More than one third 34.3\% complained of current ear problems, and 29.5\% complained of ear pain. On physical examination, $24.4 \%$ were found to have detectable ear problems, of which $42.9 \%$ had otitis, while the remainder had ceremonious occlusion of the auditory canal. There was no significant difference by gender in the prevalence of detectable ear problems.

\section{CONCLUSION:}

Overall, this paper introduces a material that can be used for further implementation in the noise reduction of high-level machinery. It describes the 
noise problem which is prevalent in today's society and also provides a way or solution to reduce the noise. The noise level reduced by Sorbothane can be of optimum level but when it is implemented in testing, the real value can be obtained. Many materials that contain the sound reduction property along with abrasion, tensile strength, elongation and also sometimes absorbency properties should be taken into account for this area ${ }^{[7]}$. Noise reduction is a critical area and future researchers should look into this matter seriously for the betterment of the society.

\section{REFERENCES:}

[1] Noise and Noise Control",S. Morita : To- kyo, Ohmu-sha,(1961).

[2] "The Structure of the Mill Wall and its Effect on the Control of Shuttle-weaving Noise". T. Ishida, A. Umeda. Journal of the Textile Institute 79:1, pages 53-65.(1988) .

[3] "Noise pollution and its control in textile industry",M K Talukdar,Indian Journal of Fibre \& Textile Research,(2001),

[4] "Handbook of Noise Control",C. M. Harris New York, McGraw-Hill Book Co,(1957).

[5] Y. Tokita J.Acoust. Soc. Japan : 20, 270 (1964).

[6] "Noise Generated by a Power Loom", Sadao Aso, Rikuhiro Kinoshita, Heihachi Uematsu and Kiyohumi Sasaki, Journal of the Textile Machinery Society of Japan, Transactions, 18, T13-19 (1965).

[7] "Noise and Vibration Control of Weaving Industry",Isogai , B. 37Osaka, , Japan: Textile Machinery Society of Japan, (1961).

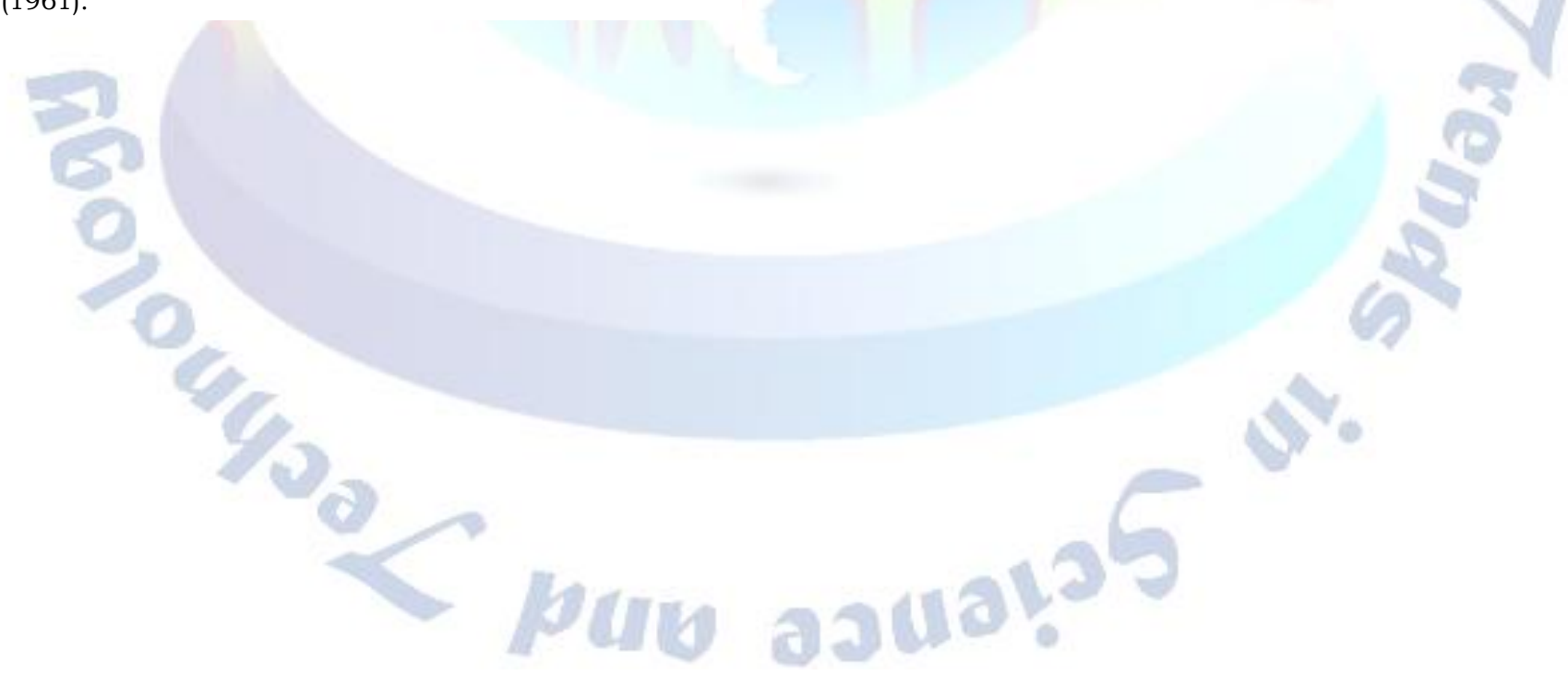

